\title{
Pre-diagnostic colonoscopies reduce cancer mortality - results from linked population- based data in South Australia
}

Ming Li $i^{*}$ DD, lan Olver ${ }^{1}$, Dorothy Keefe ${ }^{2}$, Carol Holden ${ }^{3}$, Dan Worthley ${ }^{3}$, Timothy Price ${ }^{4}$, Christos Karapetis ${ }^{5}$, Caroline Miller ${ }^{3}$, Kate Powell ${ }^{3}$, Dianne Buranyi-Trevarton ${ }^{2}$, Kellie Fusco $^{1}$ and David Roder ${ }^{1}$

\begin{abstract}
Background: To investigate the association between pre-diagnostic colonoscopy and colorectal cancer mortality in South Australia.

Methods: Colonoscopy histories were obtained for colorectal cancer patients diagnosed in 2003-2013 using linked Medical Benefits Schedule (MBS) claims, hospital-inpatient and cancer-registry data. Colonoscopy histories included the year of colonoscopy, numbers of examinations, and the time from first colonoscopy to diagnosis. Histories of multiple exposures to colonoscopies, and exposures of greater than a year from initial colonoscopy to diagnosis, were regarded as indicators of screening or surveillance activity. Colonoscopies occurring within one year of diagnosis were regarded as more likely to be a response to cancer symptoms than those occurring $\geq 1$ year before diagnosis. Associations between colonoscopy history and post-diagnostic survival were analysed using sub-hazard ratios (SHRs) from competing risk regression adjusted for socio-demographic and cancer characteristics.

Results: Having pre-diagnostic colonoscopy was associated with an unadjusted reduction in risk of colorectal cancer death of $17 \%$ (SHR: $0.83,95 \% \mathrm{Cl} 0.78-0.89$ ). After adjusting for time period and sociodemographic characteristics, the risk of colorectal cancer death reduced by $17 \%$ for one pre-diagnostic colonoscopy examination; $27 \%$ for two pre-diagnostic colonoscopy examinations; and 45\% for three or more pre-diagnostic colonoscopy examinations. Those with a time of over one year from first colonoscopy in the study window to diagnosis, when compared with less than one year, had a 17\% lower risk of colorectal cancer death in this adjusted analysis. These reductions were substantially reduced or eliminated when also adjusting for less advanced stage.
\end{abstract}

Conclusions: Pre-diagnostic colonoscopy, and more so, multiple colonoscopies and first colonoscopy occurring over one year from initial colonoscopy to diagnosis, were associated with longer survival post diagnosis. This was largely explained by less advanced cancer stage at the time of diagnosis.

Keywords: Colonoscopy history, Colorectal cancer death, Competing risk analysis, South Australia, Linked inpatient and medical benefits schedule data

\footnotetext{
* Correspondence: ming.li@unisa.edu.au

${ }^{1}$ Cancer Research Institute, University of South Australia, Adelaide, Australia

Full list of author information is available at the end of the article
}

(c) The Author(s). 2019 Open Access This article is distributed under the terms of the Creative Commons Attribution 4.0 International License (http://creativecommons.org/licenses/by/4.0/), which permits unrestricted use, distribution, and reproduction in any medium, provided you give appropriate credit to the original author(s) and the source, provide a link to the Creative Commons license, and indicate if changes were made. The Creative Commons Public Domain Dedication waiver (http://creativecommons.org/publicdomain/zero/1.0/) applies to the data made available in this article, unless otherwise stated. 


\section{Background}

Australian cancer registry data indicate that colorectal cancer has the third highest recorded incidence of cancer after prostate and breast cancer [1]. Efforts to improve outcomes of colorectal cancer have been beneficial with survival gains attributed to advances in adjuvant therapy, increased surgical specialisation, and earlier detection $[2,3]$.

The National Bowel Cancer Screening Program (NBCSP) commenced in 2006 with a staggered introduction occurring by age during 2006-2020 [2]. A data linkage study found that bowel cancers diagnosed through the NBCSP were more likely to present at an earlier stage at diagnosis than other bowel cancers [2].

The NBCSP aims to reduce morbidity and mortality from bowel cancer through biennial screening by faecal occult blood testing (FOBT) of the population aged 5074 years, and with follow-up colonoscopy of FOBTpositive cases [2-4]. The NBCSP follows Australian Clinical Practice Guidelines for the Prevention, Early Detection and Management of Colorectal Cancer, which is endorsed by the National Health and Medical Research Council [4].

Despite an absence of randomized trial evidence of effects of colonoscopy screening on colorectal cancer mortality, the US Preventive Services Taskforce supports use of FOBT with colonoscopy as a primary screening test [5]. Other evidence of mortality reductions exists from earlier detection. Trial results indicate that screening by FOBT can reduce colorectal cancer mortality [6] and evidence exists that sigmoidoscopy examinations can reduce colorectal cancer mortality [7-10].

This is the first study using linked cancer registry and administrative data to explore effects of pre-diagnostic colonoscopy screening on colorectal cancer mortality in South Australia and whether mortality benefits are occurring as expected from the US Preventive Services Taskforce recommendations [5]. Its focus is evaluation of translation of research evidence into practice rather than contributing to the scientific basis for pre-diagnostic colonoscopy.

While earlier linked data have shown sociodemographic disparities in survival and surgical mortality for colorectal cancer $[11,12]$, research into effects of colonoscopy surveillance on colorectal cancer outcomes has been limited in Australia. This study uses colorectal cancer data for South Australia to examine associations between pre-diagnostic colonoscopy and survival.

\section{Methods}

\section{Data sources and linkage}

Data on colorectal cancers (ICD10 C18-20) diagnosed between January 2003 and December 2013 were extracted from the South Australian Cancer Registry (SACR), which collects under legal mandate data on all invasive colorectal cancer diagnoses in South Australian residents [11, 12]. Data coverage is virtually complete and data fields collected include cancer topography and morphology, age at diagnosis, date of diagnosis, country of birth, Indigenous status, postcode-derived relative socioeconomic disadvantage and geographic remoteness, local health network of residence, and death date and cause [11-16].

To these data were added Australian Clinico-Pathological Staging (ACPS) data derived from SACR records [17]. Pretreatment clinical staging was used, which included evidence from MDT reports, diagnostic biopsies and imaging reports (including data from ultrasound examinations, MRI of the pelvis, CT scans, and PET), and reports of endoscopic and other physical examination [17].

While screening participation and FOBT outcome data collected by NBCSP are close to complete, reporting of NBCSP colonoscopies is not legally mandated and is incomplete [2]. We have therefore used integrated hospital inpatient data and Medical Benefits Schedule (MBS) claims to gain a better estimate of the use of pre-diagnostic colonoscopies.

Hospital inpatient data for 2002-2013 were obtained from the Integrated South Australian Activity Collection (ISAAC), including year of admission and clinical procedure codes. MBS claims data on endoscopies were also extracted for 2000-2013, including dates of services and item numbers. Inpatient data included treatment and procedures for all hospitalized patients while MBS covered tests and examinations by doctors to diagnose and treat illnesses for out-of-hospital services. Population coverage is virtually complete. MBS is a universal health insurance scheme that covers colonoscopy examinations undertaken in the private health sector whereas hospital inpatient data cover colonoscopies undertaken on inpatients.

Probabilistic and deterministic linkage methods were used to link SACR, ISAAC inpatient and MBS claims data. Data linkage between the SACR and ISAAC collections was undertaken by SANT DataLink through probabilistic matching using name, sex, date of birth and address. Data linkage between these data and MBS claims data was undertaken through the Australian Institute of Health and Welfare (AIHW), using a study linkage key also derived from names, sex, dates of birth, and addresses. The process of data linkage followed the principle of separating patient identifiers from clinical content data to protect privacy.

The study was approved by the South Australia Human Research Ethics Committee (HREC/16/SAH/6), the University of South Australia's Human Research Ethics Committee (0000036128), and the Australian Institute of Health and Welfare Human Research Ethics Committee (EO2016/4/317). 


\section{Data coding and classification}

ISAAC procedure codes used for colonoscopy were $3,209,000,3,209,001,3,209,002$ and 3,209,300, whereas the corresponding MBS codes were 32,090 for fibreoptic colonoscopy of the colon beyond the hepatic flexure without biopsy; and 32,093 for colonoscopy examinations with the removal of polyps. History of pre-diagnostic colonoscopy in the study window was classified by: (a) number of colonoscopy examinations (none, one, two, $\geq 3$ ), and (b) time from colonoscopy to cancer diagnosis $(<1$ year, $\geq 1$ year $)$. For the purposes of this study methodology, we assumed that colonoscopies undertaken in response to cancer symptoms were more likely to have occurred in the oneyear period immediately preceding diagnosis, whereas screening and surveillance colonoscopies were more likely to have occurred prior to this one-year period [4].

Age at diagnosis was classified as $<60,60-69$ or $70+$ years; country of birth as country/region using the Standard Australia Classification of Countries [14]; socioeconomic disadvantage using the Index of Relative Socioeconomic Disadvantage (SEIFA 2006) based on Statistical Local Areas grouped into quintiles (the first quintile being the most disadvantage and the 5th quintile being the least disadvantaged) [15]; and remoteness of residence based on Statistical Local Areas aggregated according to the 2006 Australian Standard Geographical Classification (ASGC) as major city, inner regional, outer regional, remote, or very remote [16]. Local health networks (LHNs) of residence were determined by data custodians from residential postcodes and classified as Country Health South Australia, Central Adelaide, Southern Adelaide, and Northern Adelaide.

Degree of spread of cancer at diagnosis was obtained from Australian Clinico-Pathological Staging (ACPS) and summarised as localised, regional or distant metastases using Tumour-lymph Node- Metastasised (TNM) criteria which were broadly equivalent to SEER summary staging criteria [17].

\section{Statistical analysis}

Pre-diagnostic colonoscopy examinations were analysed by population and cancer characteristic using the chisquare test or other non-parametric tests depending on variable distribution. Deaths were classified: as due to colorectal cancer or another cause, based on SACR records; and by time to death (irrespective of cause) between diagnosis and death. For those alive at the end of the study follow-up period (December 31st, 2013), the follow-up period was the duration between diagnosis and this end date.

The association of colonoscopy with risk of colorectal cancer-specific death was analysed using competing risk regression models (STATA module "stcrreg"). Deaths from causes other than colorectal cancer were regarded as the competing risk. The association was firstly adjusted for population characteristics and colonoscopy time period (Model 1); and then in addition for cancer stage (Model 2). Risk of colorectal cancer mortality was analysed by colonoscopy history (i.e., number of colonoscopies, period and follow-up time from first colonoscopy to diagnosis). Reference categories for the dummy variables used in analyses were the first categories listed in Tables 1, 2, 3.

Analyses were conducted using STATA 14 (STAT ACorp, College Station, Texas, USA), with statistical significance level set as $p<0.05$. All the analyses were based on complete case data.

\section{Results}

\section{Descriptive features}

Linked data for the 12,906 colorectal cancer patients diagnosed in 2003-2013 indicated the following descriptive features: a mean age of 70.2 years (SD 13.0); $56 \%$ aged $70+$ years at diagnosis; $54 \%$ male; $94 \%$ born in Australia, New Zealand or Europe; 0.3\% Indigenous (Aboriginal or Torres Strait Islander); 71\% resident in a major city (metropolitan Adelaide); a quarter from the most socio-economically disadvantaged areas (quintile 1 ); and $46 \%$ of those with a recorded stage having localized disease (Table 1).

\section{Pre-diagnostic colonoscopy}

Of the 12,906 colorectal cancer patients, $37 \%$ had prediagnostic colonoscopy. Compared to those without this history, they were older at diagnosis; more likely to have been born in Australia, New Zealand or Europe; and more likely to live in outer regional or remote, and more socioeconomically disadvantaged areas. They were also more likely to have a localized cancer and to experience a higher survival at five years post diagnosis (Table 1).

Of those having a pre-diagnostic colonoscopy, $76 \%$ had one colonoscopy, $16 \%$ had two, and $8 \%$ had three or more colonoscopies (a maximum of 11). They comprised 3048 patients having 3583 colonoscopies recorded in hospital data, and 1963 patients having 2969 colonoscopies recorded in MBS claims (note: 299 of these patients had colonoscopies recorded in both the hospital and MBS data).

The mean time from initial colonoscopy to cancer diagnosis was 1.5 years, with an interquartile range of 0 2.5 years. Of the 4712 having a pre-diagnostic colonoscopy, $63 \%$ were diagnosed with colorectal cancer within one year of this initial colonoscopy examination and $37 \%$ more than one year after this initial pre-diagnostic colonoscopy.

Compared with those having only one colonoscopy, those having more than one were significantly $(p<0.05)$ more likely: (a) to be aged 60-79 years; (b) to have been 
Table 1 Population and cancer characteristics by pre-diagnostic colonoscopy among colorectal cancer patients registered in 20032013, South Australia $(n=12,906)$

\begin{tabular}{|c|c|c|c|c|}
\hline & No colonoscopy $n=8194$ & Colonoscopy $n=4712$ & Total $n=12,906$ & $P$ value \\
\hline Mean age (SD) at diagnosis (years) & $69.4(13.5)$ & $71.5(12.0)$ & $70.2(13.0)$ & $<0.001$ \\
\hline Age at diagnosis (years) & & & & $<0.001^{* *}$ \\
\hline$<60$ & $1843(22.5 \%)$ & $764(16.2 \%)$ & 2607 (20.0\%) & \\
\hline $60-69$ & $2025(24.7 \%)$ & 1067 (22.7\%) & 3092 (24.0\%) & \\
\hline 70-79 & $2191(26.8 \%)$ & $1554(33.0 \%)$ & $3745(29.0 \%)$ & \\
\hline$\geq 80$ & $2129(26.0 \%)$ & $1326(28.2 \%)$ & 3455 (26.8\%) & \\
\hline Sex & & & & $0.043^{*}$ \\
\hline Male & $4365(54.7 \%)$ & 2447 (52.8\%) & $6812(54.0 \%)$ & \\
\hline Female & $3621(45.3 \%)$ & $2188(47.1 \%)$ & $5809(46.0 \%)$ & \\
\hline Country of birth & & & & $<0.001^{*}$ \\
\hline Australia \& NZ & $5486(68.9 \%)$ & $3206(70.2 \%)$ & $8692(69.4 \%)$ & \\
\hline Europe & $1931(24.3 \%)$ & 1153 (25.3\%) & $3084(24.6 \%)$ & \\
\hline North Africa/Middle East & $18(0.2 \%)$ & $16(0.4 \%)$ & $34(0.3 \%)$ & \\
\hline Asia \& Pacific Islands & $114(1.5 \%)$ & $52(1.2 \%)$ & $166(1.4 \%)$ & \\
\hline Americas & $31(0.4 \%)$ & $8(0.2 \%)$ & $39(0.3 \%)$ & \\
\hline Africa (other) & $370(4.7 \%)$ & $130(2.9 \%)$ & $500(4.0 \%)$ & \\
\hline Indigenous & & & & $0.100^{*}$ \\
\hline No & 7596 (99.8\%) & 4476 (99.6\%) & $12,072(99.7 \%)$ & \\
\hline Yes & $19(0.3 \%)$ & $19(0.4 \%)$ & $38(0.3 \%)$ & \\
\hline Remoteness & & & & $<0.001^{* *}$ \\
\hline Major city & $6022(73.5 \%)$ & 3174 (67.4\%) & 9196 (71.3\%) & \\
\hline Inner regional & $893(10.9 \%)$ & $508(10.8 \%)$ & $1401(10.9 \%)$ & \\
\hline Outer regional and remote & $1278(15.6 \%)$ & $1029(21.8 \%)$ & $2307(17.9 \%)$ & \\
\hline SES disadvantage quintile & & & & $<0.001^{* *}$ \\
\hline 1st (most disadvantage) & $1884(23.0 \%)$ & $1327(28.2 \%)$ & 3211 (24.9\%) & \\
\hline 2nd & $1813(22.1 \%)$ & $1058(22.5 \%)$ & $2871(22.3 \%)$ & \\
\hline $3 r d$ & $1545(18.9)$ & $936(19.9 \%)$ & $2481(19.3 \%)$ & \\
\hline 4th & $1615(19.7 \%)$ & $809(17.2 \%)$ & $2424(18.8 \%)$ & \\
\hline 5th (least disadvantage) & $1332(16.3 \%)$ & $572(12.2 \%)$ & $1904(14.8 \%)$ & \\
\hline Local Health Network & & & & $<0.001^{*}$ \\
\hline Country Health SA & $2424(29.6 \%)$ & $1689(35.9 \%)$ & $4113(31.9 \%)$ & \\
\hline Central Adelaide & $2507(30.6 \%)$ & $1269(26.9 \%)$ & $3776(29.3 \%)$ & \\
\hline Northern Adelaide & $1628(19.9 \%)$ & $872(18.5 \%)$ & $2500(19.4 \%)$ & \\
\hline Southern Adelaide & $1634(19.9 \%)$ & $880(18.7 \%)$ & $2514(19.5 \%)$ & \\
\hline Median (IQR) of years survived on Dec 31st 2013 & $2.7(0.9-5.5)$ & $3.0(1.1-6.3)$ & $2.8(1.0-5.7)$ & $<0.001$ \\
\hline Cancer staging (max spread) & & & & $<0.001^{* *}$ \\
\hline Local & $2001(42.9 \%)$ & $1479(49.8 \%)$ & $3480(45.6 \%)$ & \\
\hline Regional & $1556(33.4 \%)$ & $897(30.2 \%)$ & $2453(32.1 \%)$ & \\
\hline Distant & $1108(23.8 \%)$ & $595(20.0 \%)$ & $1703(22.3 \%)$ & \\
\hline$\%$ cancer-specific survival by time from diagnosis & & & & $<0.001^{* * *}$ \\
\hline At 1 year & $84.5 \%$ & $86.8 \%$ & $85.5 \%$ & \\
\hline At 3 year & $71.0 \%$ & $74.6 \%$ & $72.3 \%$ & \\
\hline At 5 year & $64.6 \%$ & $69.4 \%$ & $66.0 \%$ & \\
\hline
\end{tabular}


born in Australia or New Zealand; (c) to live in socioeconomically advantaged areas; (d) to be diagnosed more than one year after initial colonoscopy; (e) to be diagnosed with localized as opposed to more advanced tumours; and (f) to have longer survival (Additional file 1: Table S1).

\section{Cancer survival}

Of the 12,906 patients with colorectal cancer, $29 \%$ died from their cancer and 13\% of other causes in the followup period to December 31st, 2013. The mean survival time from diagnosis was 2.8 years (IQR 1.0-5.7).

Unadjusted sub-hazards ratios (using other deaths as competing risks) were: (a) higher for ages $70+$ years at diagnosis than $<60$ years; (b) lower for patients born in Africa (other) than Australia and New Zealand; (c) higher for Indigenous than non-Indigenous patients; (d) higher for residents living in outer regional and remote than major city areas; (e) lower for residents of less disadvantaged areas; (f) lower for localized cancers; and (g) lower for place of residence in the Southern Adelaide than the Country Health South Australia Network (Table 2).

Patients having a pre-diagnostic colonoscopy were at a lower unadjusted risk of colorectal cancer death (SHR 0.83, 95\% CI 0.78-0.89) compared with those not having a colonoscopy (Table 2). Among those having a prediagnostic colonoscopy, the unadjusted risk was lower in patients having a pre-diagnosis colonoscopy more than one year before diagnosis (SHR 0.82 (0.73-0.93), and compared with those having one pre-diagnostic colonoscopy, potentially lower in those having two (SHR 0.90 $(0.77-1.05))$ and lower in those having $3+$ pre-diagnostic colonoscopies (SHR 0.63 (0.49-0.81)) (Table 2).

When adjusting for socio-demographic characteristics and initial colonoscopy year, the risk of colorectal cancer death reduced by: (a) 17\% when having one colonoscopy (SHR 0.83, 95\% CI 0.77-0.90); (b) 27\% when having two colonoscopies (SHR 0.73, 95\% CI 0.63-0.89); and (c) $45 \%$ when having three or more colonoscopies (SHR 0.55, 95\% CI 0.43-0.70) (Table 3: Model 1). The risk also reduced in this analysis when the time from initial colonoscopy to diagnosis was one year or more (SHR 0.83, 95\% CI 0.73-0.94) compared with less than one year (Table 3: Model 1). Adjusting in addition for stage reduced the scale of reductions in risk of colorectal cancer death to $32 \%$ for three or more colonoscopies (SHR $0.68,95 \%$ CI $0.53-0.86)$ and largely eliminated the reduction in risk where the initial colonoscopy occurred more than one year pre-diagnosis (SHR 0.97, 95\% CI 0.85-1.10) (Table 3: Model 2).

Other factors found in the final full model to be associated with an increased risk of colorectal cancer death included: older age (SHR for aged 70-79 years of 1.36,
95\% CI 1.22-1.47, and for aged 80 + years of 1.92 , 95\% CI 1.75-2.12 compared with < 60 years); being Indigenous (SHR 1.57, 95\% CI 1.07-2.31); and having an advanced cancer stage when compared with localized stage (SHR for regional stage of 3.05, 95\% CI 2.71-3.43 and for distant stage of $11.16,95 \%$ CI 9.99-12.46). By comparison, socioeconomic advantage was associated with a lower risk of colorectal cancer death (SHR for the most advantaged of $0.85,95 \%$ CI $0.76-0.95$ compared with most disadvantaged).

Model 1: adjusted for age, sex, country of birth, Indigenous status, residential remoteness, socioeconomic status, local health network, initial colonoscopy year; Model 2: adjusted for the same characteristics as Model 1 , plus stage.

Notably sex, country of birth, residential remoteness, and local health district were no longer associated with risk of colorectal cancer death in the multivariate competing risk regression analysis $(p>0.050)$. Also, the association between pre-diagnostic colonoscopy and colorectal cancer death in the fully adjusted model was not different among subgroups classified by age at diagnosis, Indigenous status and socio-economic status $(p>0.050)$.

\section{Discussion}

Results indicate that having a pre-diagnostic colonoscopy was associated with a $17 \%$ lower risk of colorectal cancer death. After adjusting for sociodemographic and cancer characteristics, the risk of colorectal cancer death reduced step-wise with increasing numbers of colonoscopy examinations to $45 \%$ for three or more compared with no colonoscopy. Also, a $17 \%$ reduction applied when the time to diagnosis from initial colonoscopy was $\geq 1$ year compared with $<1$ year.

Stage appeared to mediate much of these reductions such that adjusting in addition for stage: [1] reduced the scale of reduction with increased number of colonoscopies; and [2] largely eliminated the increased reduction associated with a longer time from colonoscopy to diagnosis.

Our results indicate a protective benefit of pre-diagnostic colonoscopy which is consistent with results from the USA Nurses' Health Study and Health Professionals Follow-up [18] and the more recent case-control study of Veterans Affairs patients [19]. Effect sizes were larger in the USA data, potentially reflecting differences in reasons for colonoscopies. We consider that the use of colonoscopy in this South Australian study would have included more symptomatic cases leading to a smaller reduction in risk of colorectal from prediagnostic colonoscopy. Other factors that could have affected the reduction in risk included sample selection and follow-up period [20-22]. 
Table 2 Characteristics associated with colorectal cancer mortality post diagnosis and colonoscopy history (deaths by December 31st, 2013, registered in 2003-2013, South Australia $(n=12,906)$

\begin{tabular}{|c|c|c|c|c|}
\hline Characteristics & Colorectal cancer death $n=3771$ & Other death $n=1621$ & Alive $n=7514$ & Unadjusted SHR $(95 \% \mathrm{Cl}){ }^{*}$ \\
\hline Mean age (SD) at diagnosis (years) & $72.2(13.2)$ & $78.3(10.0)$ & $67.4(12.6)$ & $1.13(1.11-1.16)$ \\
\hline \multicolumn{5}{|l|}{ Age at diagnosis (years) } \\
\hline$<60$ & $652(17.3 \%)$ & $68(4.2 \%)$ & $1887(25.1 \%)$ & 1.00 \\
\hline $60-69$ & $781(20.8 \%)$ & $193(11.9 \%)$ & $2118(28.2 \%)$ & $1.00(0.91-1.11)$ \\
\hline 70-79 & $1056(28.0 \%)$ & $495(30.5 \%)$ & $2194(29.2 \%)$ & $1.13(1.03-1.25)$ \\
\hline$\geq 80$ & $1280(34.0 \%)$ & $865(53.4 \%)$ & $1310(17.5 \%)$ & $1.70(1.55-1.87)$ \\
\hline \multicolumn{5}{|l|}{ Sex } \\
\hline Male & $2014(53.6 \%)$ & $917(56.6 \%)$ & $3881(53.6 \%)$ & 1.00 \\
\hline Female & $1745(46.4)$ & $703(43.4 \%)$ & 3361 (46.4\%) & $1.03(0.97-1.10)$ \\
\hline \multicolumn{5}{|l|}{ Country of birth } \\
\hline Australia \& NZ & $2599(71.1 \%)$ & $1113(71.1 \%)$ & $4980(68.3 \%)$ & 1.0 \\
\hline Europe & $957(26.2 \%)$ & $427(27.3 \%)$ & $1700(23.3 \%)$ & $1.03(0.96-1.11)$ \\
\hline North Africa/ Middle East & $10(0.3 \%)$ & $2(0.1 \%)$ & $22(0.3 \%)$ & $0.99(0.54-1.82)$ \\
\hline Asia and Pacific Islands & $47(1.2 \%)$ & $15(1.0 \%)$ & $106(1.5 \%)$ & $0.87(0.66-1.15)$ \\
\hline Americas & $14(0.4 \%)$ & $2(0.1 \%)$ & $23(0.3 \%)$ & $1.35(0.80-2.28)$ \\
\hline Africa (other) & $32(0.9 \%)$ & $7(0.5 \%)$ & $461(6.3 \%)$ & $0.66(0.46-0.95)$ \\
\hline \multicolumn{5}{|l|}{ Indigenous } \\
\hline No & 3653 (99.5\%) & 1576 (99.9\%) & $6843(99.7 \%)$ & 1.00 \\
\hline Yes & $17(0.5 \%)$ & $2(0.1 \%)$ & $19(0.3 \%)$ & $1.85(1.12-3.05)$ \\
\hline \multicolumn{5}{|l|}{ Remoteness } \\
\hline Major city & $2663(70.6 \%)$ & 1199 (74.0\%) & $5334(71.0 \%)$ & 1.00 \\
\hline Inner regional & $386(10.2 \%)$ & $156(9.6 \%)$ & $859(11.4 \%)$ & $0.95(0.85-1.05)$ \\
\hline Outer regional and remote & $722(19.2 \%)$ & $266(16.4 \%)$ & $1319(17.6 \%)$ & $1.11(1.02-1.20)$ \\
\hline \multicolumn{5}{|l|}{ SES disadvantage quintile } \\
\hline 1st (most disadvantage) & $1020(27.1 \%)$ & $406(25.1 \%)$ & $1785(23.8 \%)$ & 1.00 \\
\hline 2nd & $838(22.3 \%)$ & $346(21.4 \%)$ & 1687 (22.5\%) & $0.92(0.84-1.00)$ \\
\hline $3 r d$ & $689(18.3 \%)$ & $365(22.5 \%)$ & $1427(19.0 \%)$ & $0.84(0.77-0.93)$ \\
\hline 4th & $699(18.6 \%)$ & $283(17.5 \%)$ & $1442(19.2 \%)$ & $0.89(0.81-0.98)$ \\
\hline 5th (least disadvantage) & $519(13.8 \%)$ & $220(13.6 \%)$ & 1165 (15.5\%) & $0.82(0.73-0.91)$ \\
\hline \multicolumn{5}{|l|}{ Local Health Network code } \\
\hline Country Health SA & $1211(32.1 \%)$ & $474(29.2 \%)$ & $2428(32.3 \%)$ & 1.00 \\
\hline Central Adelaide & $1122(29.8 \%)$ & $527(32.5 \%)$ & $2127(28.3 \%)$ & $1.00(0.92-1.09)$ \\
\hline Northern Adelaide & $755(20.0 \%)$ & $273(16.8 \%)$ & $1472(19.6 \%)$ & $1.04(0.95-1.14)$ \\
\hline Southern Adelaide & $683(18.1 \%)$ & $347(21.4 \%)$ & $1484(19.8 \%)$ & $0.90(0.82-0.98)$ \\
\hline Years survived on Dec 31st 2013 Median (IQR) & $2.8(0.9-5.1)$ & $2.8(0.9-5.1)$ & $4.4(1.9-7.2)$ & \\
\hline \multicolumn{5}{|l|}{ Cancer staging (max spread) } \\
\hline Local & $439(18.1 \%)$ & $547(59.6 . \%)$ & $2494(58.2 \%)$ & 1.00 \\
\hline Regional & $783(32.2 \%)$ & $277(30.2 \%)$ & $1393(32.5 \%)$ & $3.05(2.72-3.42)$ \\
\hline Distant & $1208(49.7 \%)$ & $94(10.2 \%)$ & $401(9.4 \%)$ & $11.41(10.24-12.71)$ \\
\hline \multicolumn{5}{|l|}{ Pre-diagnosis colonoscopy } \\
\hline No & $2505(66.4 \%)$ & $899(55.5 \%)$ & $4790(63.8 \%)$ & 1.00 \\
\hline Yes & 1266 (33.6\%) & $722(44.5 \%)$ & $2724(36.3 \%)$ & $0.83(0.78-0.89)$ \\
\hline
\end{tabular}

Follow-up time from initial colonoscopy to diagnosis 
Table 2 Characteristics associated with colorectal cancer mortality post diagnosis and colonoscopy history (deaths by December 31st, 2013, registered in 2003-2013, South Australia $(n=12,906)$ (Continued)

\begin{tabular}{lllll}
\hline Characteristics & Colorectal cancer death $n=3771$ & Other death $n=1621$ & Alive $n=7514$ & Unadjusted SHR (95\% Cl) * \\
\hline $\begin{array}{c}\text { 1 year } \\
>=1 \text { year }\end{array}$ & $883(69.8 \%)$ & $529(73.3 \%)$ & $1577(57.9 \%)$ & 1.00 \\
Pre-diagnostic colonoscopy examinations & $383(30.3 \%)$ & $193(26.7 \%)$ & $1147(42.1 \%)$ & $0.82(0.73-0.93)$ \\
One & $1023(80.8 \%)$ & & & \\
Two & $179(14.1 \%)$ & $560(77.6 \%)$ & $1991(73.1 \%)$ & 1.00 \\
$>=3$ & $64(5.1 \%)$ & $119(16.5 \%)$ & $446(16.4 \%)$ & $0.90(0.77-1.05)$ \\
\end{tabular}

*SHR (sub Hazard ratio) from competing risk survival analysis with death other than colorectal cancer as competing risk in the univariate model; cell totals < overall totals due to missing records

Our results support the conclusion that pre-diagnostic colonoscopy in South Australia lowers the risk of colorectal cancer death, largely by effecting an earlier stage at diagnosis. The association of a lower risk with multiple colonoscopy examinations and longer duration from initial colonoscopy to diagnosis (1+ years) is suggestive of a protective benefit from colonoscopy used in response to positive FOBT screening results in the average risk population or to surveillance of high-risk patients drawn from primary health care settings. By comparison, colonoscopy in response to colorectal cancer symptoms likely would have occurred closer to diagnosis (i.e., within a year).

Results support the importance of adherence to Australian clinical guidelines on early detection of colorectal cancer [4]. To reduce colorectal cancer mortality further, participation in the National Bowel Screening Program should be increased through promotion of awareness among primary health care practitioners and the Australian public. Present levels of FOBT screening are low, with a 2 -year participation rate of only $41 \%$ between January 2015 and December 2016. Moreover, of those having a positive FOBT result, only $68 \%$ were reported to have had follow-up diagnostic assessment [23].

Residents of remote and low socio-economic areas, and Indigenous screeners, have lower rates of colonoscopy and longer durations to colonoscopy assessment following a FOBT, despite elevations in positive FOBT results [23]. Operational research into the most effective strategies for addressing these disparities is needed.

The study also has limitations. We could not determine the purpose of colonoscopies from the direct recordings available to us through bowel screening registries, MBS and inpatient datasets and we inferred the purpose of colonoscopies from the duration from the initial colonoscopy to diagnosis, and evidence of multiple colonoscopies. We recognize that this approach would have lacked the accuracy possible though direct reporting, had it been available. Nonetheless, the data indicate a lower risk of colorectal cancer death when the initial colonoscopy was over a year ago, and where there were multiple colonoscopy examinations (both of which were considered to be indicative of screening or surveillance). The association of earlier colonoscopies with reduced deaths was largely explained by earlier stage, which we interpret as a screening or surveillance benefit.

In addition, potential for misclassification due to the limited time window from inpatient data to extract pre-diagnostic colonoscopy also was a limitation. Specifically, the time period to capture MBS-funded colonoscopy data was from 2000, which was $>2$ years before the earliest diagnosis of colorectal cancer cases in the study window (2003-2013) and longer than the 2-year screening interval recommended for the national bowel cancer screening program (NBCSP). An equivalent window was not available from public

Table 3 Adjusted sub-hazard ratios $(95 \% \mathrm{Cl}$ ) of colorectal cancer death with history of pre-diagnostic colonoscopy among patients diagnosed in 2003-2013*

\begin{tabular}{|c|c|c|c|c|c|}
\hline & No. patient & Person time (person year) & No. Event & Model 1 & Model 2 \\
\hline \multicolumn{6}{|c|}{ Pre-diagnostic colonoscopy examinations } \\
\hline 0 & 8194 & $28,653.08$ & 2505 & 1.00 & \\
\hline 1 & 3574 & $14,305.83$ & 1023 & $0.83(0.77-0.90)$ & $0.85(0.79-0.91)$ \\
\hline 2 & 744 & 2600.52 & 179 & $0.73(0.63-0.89)$ & $0.81(0.70-0.95)$ \\
\hline$>=3$ & 394 & 1226.19 & 64 & $0.55(0.43-0.70)$ & $0.68(0.53-0.86)$ \\
\hline \multicolumn{6}{|c|}{ Time from initial colonoscopy to diagnosis } \\
\hline$<1$ year & 2989 & $12,481.72$ & 883 & 1.00 & 1.00 \\
\hline$>=1$ year & 1723 & 5650.82 & 383 & $0.83(0.73-0.94)$ & $0.97(0.85-1.10)$ \\
\hline
\end{tabular}

*Sub hazard ratios (SHR) from competing risk survival regression model 
hospital data for pre-diagnostic colonoscopies in 20002001 (i.e., a period $>1$ year before the study window). Although we would expect a small proportion of colonoscopies with negative results occurring $>1$ year before diagnosis to have been missed for this reason, we think it unlikely that this would have greatly affected the results. It would not have affected the size of the group with colonoscopies occurring $<1$ year before diagnosis.

Another limitation was the low numbers of cases with $1+$ years occurring between initial colonoscopy and diagnosis, limiting opportunities for further sub-classification of this group (note: the average duration from initial colonoscopy to diagnosis was 1.5 years, with an interquartile range of $0-2.5$ years). However, we consider that $1+$ years was a reasonable cut-off to separate colonoscopies that were more likely to have been be undertaken in response to cancer symptoms from those more likely to have been for screening or surveillance.

Incomplete staging data were also a key limitation. This was due to limited coverage of staging data by the electronic clinical information systems available to us. While this may have led to selection bias, we see no obvious reason for this to have occurred. Predictably these data showed a positive association of colonoscopy history with lower spread of the cancer at diagnosis. We consider that presenting these data in the context of this limitation in stage completeness is justified.

Finally, potential confounding effects on colorectal cancer mortality outcomes from differences in prevalence of comorbid conditions and related risk behaviours (including effects of smoking, alcohol drinking, physical activity, obesity, diabetes, and medication use) could not be investigated with the data available [24].

\section{Conclusions}

Having a pre-diagnostic colonoscopy is associated with a reduced risk of colorectal cancer death in South Australia. This relationship is independent of age at diagnosis, sex, country of birth, Indigenous status, socioeconomic status, residential remoteness, local health network of residence, and initial examination period.

Among those having a pre-diagnostic colonoscopy, higher numbers of colonoscopy examinations, and longer times from first colonoscopy examination to diagnosis, are associated with a reduced risk of colorectal cancer death, irrespective of socio-demographic characteristics. This is largely due to less advanced cancers at diagnosis.

The reduced risk of colorectal cancer death with multiple colonoscopy examinations, and longer time from first colonoscopy examination, is indicative of a benefit from screening or surveillance of high-risk patients, since it would largely exclude colonoscopies undertaken close to cancer diagnosis in response to symptoms.

\section{Additional file}

Additional file 1: Table S1. Population characteristics of colorectal patients in South Australia in 2003-2013 by numbers of pre-diagnostic colonoscopies $(n=4712)$. ${ }^{*}$ One-way ANOVA for age at diagnosis, ${ }^{* *}$ Fisher's exact chi-square tests; cell totals < overall totals due to missing records. (DOCX $18 \mathrm{~kb})$

\section{Abbreviations}

ACPS: Australian Clinico-Pathological Staging; AlHW: Australian Institute of Health and Welfare; ASGC: Australian Standard Geographical Classification; CHSA: Country Health South Australia; Cl: Confidence interval; FOBT: faecal occult blood test; ICD10: International Classification of Diseases, 10th revision; IQR: Interquartile range; ISAAC: Integrated South Australian Activity Collection; LHNs: Local health networks; MBS: Medical Benefits Schedule; NBCSP: The National Bowel Cancer Screening Program; NZ: New Zealand; SACR: South Australian Cancer Registry; SD: Standard deviation;

SEIFA: Socio-Economic Indexes for Areas; SHR: Sub-distribution hazard ratio; TNM: Tumour-lymph Node- Metastasised

\section{Acknowledgements}

We thank the Australian Institute of Health and Welfare (AlHW), the SANT

DataLink team, and SA Heath for their assistance in this project.

\section{Authors' contributions}

Study concept: DR, TP, CK, IO, DK, CH, DW, ML; Study Design: DR, TP, CK, IO, DK, CH, DW, ML; Data acquisition: DR, DBT, KP; Data Analysis: ML, DR; Data interpretation: $\mathrm{ML}, \mathrm{DR}, \mathrm{TP}, \mathrm{CK}, \mathrm{IO}, \mathrm{CH}$; Manuscript writing: $\mathrm{ML}$, DR; Review of manuscript: IO, DK, CH, DW, TP, CK, CM, KP, DBT, KF; ML. All authors read and approved the final manuscript.

\section{Funding}

This project is supported by Cancer Council SA's Beat Cancer Project on behalf of its donors and the State Government of South Australia through the Department of Health in data extraction and linkage.

\section{Availability of data and materials}

The data that support the findings of this study are available from the South Australian Department for Health and Wellbeing (South Australian Cancer Registry \& Integrated South Australian Activity Collection) and the Australian Institute for Health and Welfare (Medical Benefits Schedule) but restrictions apply as conditions for data custodian and ethics approvals for the study. As a result, source data are publicly available only with approval from these bodies.

\section{Ethics approval and consent to participate}

The study was approved by the South Australia Human Research Ethics Committee (HREC/16/SAH/6), the University of South Australia's Human Research Ethics Committee (0000036128), and the Australian Institute of Health and Welfare Human Research Ethics Committee (EO2016/4/317). Individual consent to participate was waived due to the nature of administrative registry data and participant privacy is protected through the use of non-identifiable data and by only reporting aggregated data.

\section{Consent for publication}

Not applicable.

\section{Competing interests}

The authors declare that they have no competing interests.

\section{Author details}

${ }^{1}$ Cancer Research Institute, University of South Australia, Adelaide, Australia. SA Cancer Service, South Australian Department for Health and Wellbeing, Adelaide, Australia. ${ }^{3}$ South Australia Health and Medical Research Institute, Adelaide, Australia. ${ }^{4}$ Clinical Oncology Research Unit, The Queen Elizabeth Hospital, Woodville, Australia. ${ }^{5}$ Flinders Medical Centre, Flinders University, Adelaide, Australia. 
Received: 13 May 2019 Accepted: 26 August 2019

Published online: 29 August 2019

\section{References}

1. Australian Institute of Health and Welfare. Cancer in Australia 2017. Cancer series no.101. Cat. No. CAN 100: Canberra. AlHW; 2017.

2. Australian Institute of Health and Welfare. Analysis of bowel cancer outcomes from the National Bowel Cancer Screening program. Cat. No. CAN 87. Canberra: AlHW; 2014.

3. Department of Health, Australia government in consultation with the Quality Framework Working Group. National Bowel Cancer Screening Program Policy Framework. endorsed by the Standing Committee on Screening in Oct, 2016.

4. Cancer Council Australia Colorectal Cancer Guidelines Working Party. Clinical practice guidelines for the prevention, early detection and management of colorectal cancer. Short form summary of NHMRC approved recommendations. Sydney. 2017. https://wiki.cancer.org.au/australia/ Guidelines:Colorectal_cancer. Accessed 12 Nov 2018.

5. US Preventive Services Task Force. Screening for colorectal cancer: US preventive services task force recommendation statement. JAMA. 2016; 315(23):2564-75.

6. Hewitson P, Glasziou PP, Irwig L, Towler B, Watson E. Screening for colorectal cancer using the faecal occult blood test, Hemoccult. Cochrane Database of Systematic Reviews, 2007, issue 1. Art. No.: CD001216. doi: https://doi.org/10.1002/14651858.CD001216.pub2,

7. Brenner $\mathrm{H}$, Stock $\mathrm{C}$, Hoffmeister M. Effect of screening sigmoidoscopy and screening colonoscopy on colorectal cancer incidence and mortality: systematic review and meta-analysis of randomised controlled trials and observational studies. BMJ. 2014;348:g2467.

8. Holme O, Schoen RE, Senore C, Segnan N, Hoff G, Loberg M, Bretthauer M, Adami HO, Kalager M. Effectiveness of flexible sigmoidoscopy screening in men and women and different age groups: pooled analysis of randomised trials. BMJ. 2017;356:16673. https://doi.org/10.1136/bmj.i6673.

9. Cancer Council Australia Surveillance colonoscopy guidelines working party. Draft clinical practice guidelines for surveillance colonoscopy. Sydney: Cancer council Australia. 2018. https://wiki.cancer.org.au/australiawiki/index. php?oldid=183316. Accessed 26 Sept 2018.

10. Emery JD, Pirotta M, Macrae F, Walker JG, Qama A, Boussioutas A, et al. 'Why don't I need a colonoscopy?' A novel approach to communicating risks and benefits of colorectal cancer screening. Aust J of Gen Pract. 2018;47(6).

11. Beckmann KR, Bennett A, Young GP, Cole SR, Joshi R, Adams J, et al. Sociodemographic disparities in survival from colorectal cancer in South Australia: a population-wide data linkage study. BMC Health Serv Res. 2016; 20(16):24.

12. Beckmann K, Moore J, Wattchow D, Young G, Roder D. Short-term outcomes after surgical resection for colorectal cancer in South Australia. J Eval Clin Pract. 2017;23:316-24

13. Roder D, Karapetis CS, Wattchow D, Moore J, Singhal N, Joshi R, et al. Colorectal cancer treatment and survival over three decades at four major public hospitals in South Australia: trends by age and in the elderly. Eur J Cancer Care. 2016;25:753-63.

14. Australia Bureau of Statistics 2016. Standard Australian Classification of Countries (SACC). ABS Catalogue No. 1269.0. ABS, Canberra. 2017. http:// www.abs.gov.au/ausstats/abs@.nsf/mf/1269.0. Accessed 30 Apr 2018.

15. Australia Bureau of Statistics 2008. Information paper: an introduction to socio-economic indexes for areas (SEIFA) 2006. ABS catalogue no. 2039.0. ABS, Canberra. 2017. http://www.abs.gov.au/ausstats/abs@.nsf/mf/2039.0. Accessed 30 Apr 2018.

16. Australia Bureau of Statistics 2011. Australian Standard Geographical Classification (ASGC) Remoteness Area Correspondences, 2006. ABS Catalogue No.1216.0.15.003. ABS, Canberra. 2011. http://www.abs.gov.au/ ausstats/abs@.nsf/products/1216.0.15.003 2006 main+Features 2006+RA+ from+2006+SLA+Correspondence?OpenDocument\#\#221227101023994955 Accessed 30 Apr 2018.

17. Cancer Council South Australia. Staging bowel cancer. 2017. https://www. cancersa.org.au/information/a-z-index/staging-bowel-cancer. Accessed 30 Aug 2018.

18. Nishihara R, Wu K, Lochhead P, Morikawa T, Liao X, Qian ZR, et al. Longterm colorectal-cancer incidence and mortality after lower endoscopy. $\mathrm{N}$ Engl J Med. 2013;369:1095-105.
19. Kahi CJ, Pohl H, Myers LJ, Mobarek D, Robertson DJ, Imperiale TF. Colonoscopy and colorectal Cancer mortality in the veterans affairs health care system: a case-control study. Ann Intern Med. 2018;168:481-8.

20. Lauby-Secretan B, Vilahur N, Bianchini F, Guha N, Straif K. For the International Agency for Research on Cancer handbook working GroupThe IARC perspective on colorectal Cancer screening. N Engl J Med. 2018;378: $1734-40$.

21. Hadjipetrou A, Anyfantakis D, Galanakis CG, Kastanakis M, Kastanakis S. Colorectal cancer, screening and primary care: a mini literature review. World J Gastroenterol. 2017;23:6049-58.

22. Australian Institute of Health and Welfare 2018. National Bowel Cancer Screening Program: monitoring report. 2018. Cat. no. CAN 112. Canberra: AlHW. 2018. https://www.aihw.gov.au/reports/cancer-screening/nationalbowel-cancer-screening-program-2018/contents/summary. Accessed 10 Sept 2018.

23. Huxley RR, Ansary-Moghaddam A, Clifton P, Czernichow S, Parr CL, Woodward M. The impact of dietary and lifestyle risk factors on risk of colorectal cancer: a quantitative overview of the epidemiological evidence. Int J Cancer. 2009;125:171-80.

24. Carr PR, Alwers E, Bienert S, Weberpals J, Kloor M, Brenner H, et al. Lifestyle factors and risk of sporadic colorectal cancer by microsatellite instability status: a systematic review and meta-analyses. Ann Oncol. 2018:29:825-34.

\section{Publisher's Note}

Springer Nature remains neutral with regard to jurisdictional claims in published maps and institutional affiliations.

\section{Ready to submit your research? Choose BMC and benefit from:}

- fast, convenient online submission

- thorough peer review by experienced researchers in your field

- rapid publication on acceptance

- support for research data, including large and complex data types

- gold Open Access which fosters wider collaboration and increased citations

- maximum visibility for your research: over $100 \mathrm{M}$ website views per year

At BMC, research is always in progress.

Learn more biomedcentral.com/submissions 